\title{
Editorial: Trait-Based Plant Community Assembly, Ecological Restoration, and the Biocontrol of Invasive Exotic Plant Species
}

\author{
Hui Zhang ${ }^{1,2 *}$, Guangyu Wang ${ }^{3}$ and Wenxing Long ${ }^{1,2}$ \\ ${ }^{1}$ College of Forestry/Wuzhishan National Long Term Forest Ecosystem Monitoring Research Station, Hainan University, \\ Haikou, China, ${ }^{2}$ Key Laboratory of Genetics and Germplasm Innovation of Tropical Special Forest Trees and Ornamental \\ Plants (Hainan University), Ministry of Education, College of Forestry, Hainan University, Haikou, China, ${ }^{3}$ Department of \\ Forest Resource Management, University of British Columbia, Vancouver, BC, Canada
}

Keywords: ecological restoration, functional trait, community assembly, ecosystem functioning, biocontrol, trait-based restoration

\section{Editorial on the Research Topic}

Trait-Based Plant Community Assembly, Ecological Restoration, and the Biocontrol of Invasive Exotic Plant Species

Given trees' photosynthetic carbon capture ability, a large number of forest reforestation initiatives (such as the Bonn Challenge, the related AFR100, and the New York Declaration on Forests) have been established globally to limit global warming to $1.5^{\circ} \mathrm{C}$ by 2050 (The Bonn Challenge,

\section{OPEN ACCESS}

Edited and reviewed by: Orsolya Valkó,

Hungarian Academy of Science, Hungary

*Correspondence: Hui Zhang

993781@hainanu.edu.cn

Specialty section:

This article was submitted to Conservation and Restoration

Ecology,

a section of the journal

Frontiers in Ecology and Evolution

Received: 02 December 2021

Accepted: 11 January 2022

Published: 07 February 2022

Citation:

Zhang H, Wang G and Long W (2022) Editorial: Trait-Based Plant Community Assembly, Ecological Restoration, and the Biocontrol of Invasive Exotic Plant Species. Front. Ecol. Evol. 10:827397.

doi: 10.3389/fevo.2022.827397 2011; UN Climate Summit, 2014). However, the current reforestation strategies do not appear to be highly effective in restoring the degraded tropical rainforests (due to rubber plantation) into late-successional tropical rainforests (Crouzeilles et al., 2016, 2017). Global reforestation strategies are divided into active (mono-planting or mixed-planting exotic, native, and several early-successional tree species, or economically important tree species), and passive (natural regeneration) reforestations (Lamb et al., 2005; Reid et al., 2018). The standard for selecting exotic, native, yet several early-successional or economically important tree species is based on their high survival and fast-growth rates under severely degraded conditions of forests that have large open areas (Martínez-Garza and Howe, 2003; Shimamoto et al., 2018). However, the arrival and establishment of late-successional species can easily be inhibited when these species thrive in the degraded forest (Mesquita et al., 2015; Shoo et al., 2016; Karina et al., 2021). This may explain why active reforestation usually fails to achieve the same ecological restoration success as natural regeneration (Crouzeilles et al., 2017). As such, it is very important that we develop effective and efficient methods for restoring them to natural or semi-natural conditions (Dobson et al., 1997; Hobbs and Harris, 2001). The first and most important step in this process is identifying species that are most appropriate and effective for restoration applications (Brown and Amacher, 1999; Jones, 2013; Fry et al., 2014). However, this step requires a comprehensive understanding of the ecological restoration theory, including information on species' interactions, successional processes, and resource-use patterns. Because these processes differ greatly across different ecosystems, this remains an enormous challenge. As this type of information is typically lacking, the selection of candidate species for restoration purposes are typically chosen using a trial-and-error method (Rosenthal, 2003; Padilla et al., 2009; Ostertag et al., 2015).

Plant functional traits, including both intra- and interspecific variations in morphological, physiological, and phenological characteristics, are fundamental to understanding plant 
adaptations and distributions. Previously, trait-based methods have been used to quantify plant community assembly and life history strategies. These methods have also been deemed an effective way of selecting candidate species for use in ecological restoration and the biocontrol of invasive exotic plant species. Trait-based plant community assembly can also provide guidance on how to use functional traits to perform ecological restoration. Trait-based life history strategies can not only reveal how invasive exotic plant species successfully invade the native ecosystem, but also facilitate the selection of native plant species to perform biocontrol. Despite the remarkable importance of functional traits in community assembly, ecological restoration, and biocontrol of invasive exotic plant species, the original questions have not yet been fully answered. Additionally, there is still a need for the development and successful application of a specific, step-by-step procedure to guide the use of functional traits for selecting species for ecological restoration and biocontrol of invasive exotic plant species. Therefore, we organized this Research Topic to understand: (1) how to use functional traits to perform investigative ecological restoration and biocontrol of invasive exotic plant species, and (2) trait-based community assembly and life history strategies during ecological restoration. The objective of this interdisciplinary Research Topic is to bring together the current research on trait-based community assembly and life history strategies during natural and artificial restoration, trait-based ecological restoration, and the biocontrol of invasive exotic plant species.

Till now our Research Topic has received 13 manuscripts and finally published 11 papers. These papers can be summarized in the following five main directions: (1) how reduced biodiversity caused by global change and biotic invasion affect biodiversity, (2) how to use functional traits to guide the protection of endangered plant species, (3) trait-based protocol for selecting appropriate species to facilitate reforestation and prevent biotic interaction, (4) trait-based protocol for using reforestation to recover mining-induced extremely degraded forest ecosystems, and (5) the influences of reforestation on ecosystem functioning and service and how to use functional traits to further improve the related reforestation project. Their details are described as below:

Global nitrogen eutrophication, which is disrupting the intimate plant-arbuscular mycorrhizal fungi (AMF) symbiosis, can alter the diversity and physiological functions of soil AMF greatly. However, shifts of beta diversity and the intrinsic patterns of AMF community dissimilarities in response to nitrogen addition remain unclear. Based on a 7-year nitrogen addition experiment in a Qinghai-Tibet Plateau alpine meadow, $\mathrm{Lu}$ et al. discovers the mechanism of arbuscular mycorrhizal fungi (AMF) diversity change along nitrogen addition gradient, by partitioning two aspects (Simpson and Nestedness) of the overall community beta diversity. They found that nitrogen addition not only decreased AMF richness, but the overall community beta diversity also showed different patterns along and within nitrogen addition treatment. AMF community overall beta diversity along the nitrogen addition gradients was induced by the increased nestedness dissimilarity, while the variation within treatments was explained by both increased Simpson and nestedness dissimilarities, which was correlated with plant and soil environmental dissimilarities. Eutrophication of aquatic ecosystems is a serious global issue. By measuring the possible effects of deer population on nitrate concentrations in a nearby stream in a forest ecosystem in Japan. Tsuboike et al. (2021) shows that the estimated community-level NRA inside the fence was 5.6 times higher than that of the open area and the difference was greatest early in the season. The authors concluded that degradation of the understory vegetation, especially of herbaceous plants, by the deer overpopulation was one of the reasons for the increased nitrate concentration in the stream.

By using functional traits, some useful conservation methods of two endangered plant species (Horsfieldia hainanensis and Ottelia cordata) and overgrazed meadows in Qinghai Tibetan Plateau have been uncovered. For example, by quantifying the photosynthetic traits (i.e., maximum photosynthesis rate, photosynthetic pigment indicators, leaf morphological traits, and leaf water content) of $H$. hainanensis to different intensity of light transmittance (7.3-44.41\%), finds Wang et al. that $H$. hainanensis can have high potential to fulfill natural return, when letting it be exposed to $44.41 \%$ light transmittance. Similarly, by quantifying the relative importance of biological traits that were related to demographic traits (growth, survival, and reproduction) and water depth on population dynamic (abundance) of $O$. cordata, Shen et al. reveals that in the short term for avoiding the potential harm or even extinction of O. cordata, keeping appropriate water depth or transplanting O. cordata to spring should be an effective strategy. In the long run, in order to make the population recover, it was necessary to restore the degraded wetland, such as reducing water pollution and removing sludge to keep the water clean. In addition, by investigating whether the response of aboveand below-ground traits to overgrazing vary between the species of annual vs. perennial life-forms, Zhang et al. demonstrates that overgrazing resulted in large removal of perennial species at all elevations, which forced perennial species to alter their above- and below-ground traits so that they could shift from resource conservation to resource acquisition to avoid overgrazing. In contrast, overgrazing promoted annual species at all elevation, thus annual species did not make any response to overgrazing. These findings bridge the gap in the literature for the influence of annual vs. perennial life-forms on speciesspecific response at both above- and below-ground levels during overgrazing. More important, these results can facilitate selecting species for sustainable ecosystem management and restoration from overgrazing.

The general and effective protocol for selecting suitable native plant species to perform effective reforestation and biotic invasion prevention has also been attained by using functional traits. For instance, by using functional trait, a native tree species (Bombax ceiba) has been found to be a good native tree species for performing reforestation to restore highly degraded tropical forests in Hainan Island, China (Luo et al.). A general trait-based protocol has also been developed for quick and effective selection of suitable native tree species to perform reforestation and biotic invasion prevention (Wang et al.; Zhang et al.). They also use their developed trait-based protocol to successfully restore 


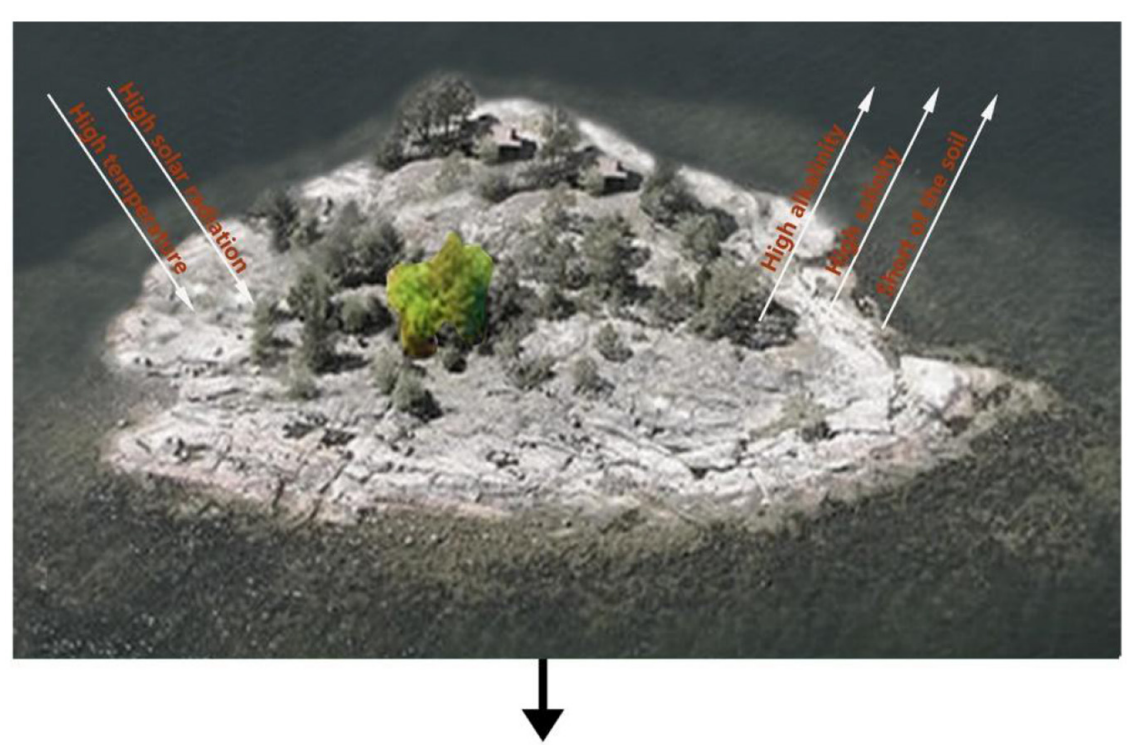

Functional Traits
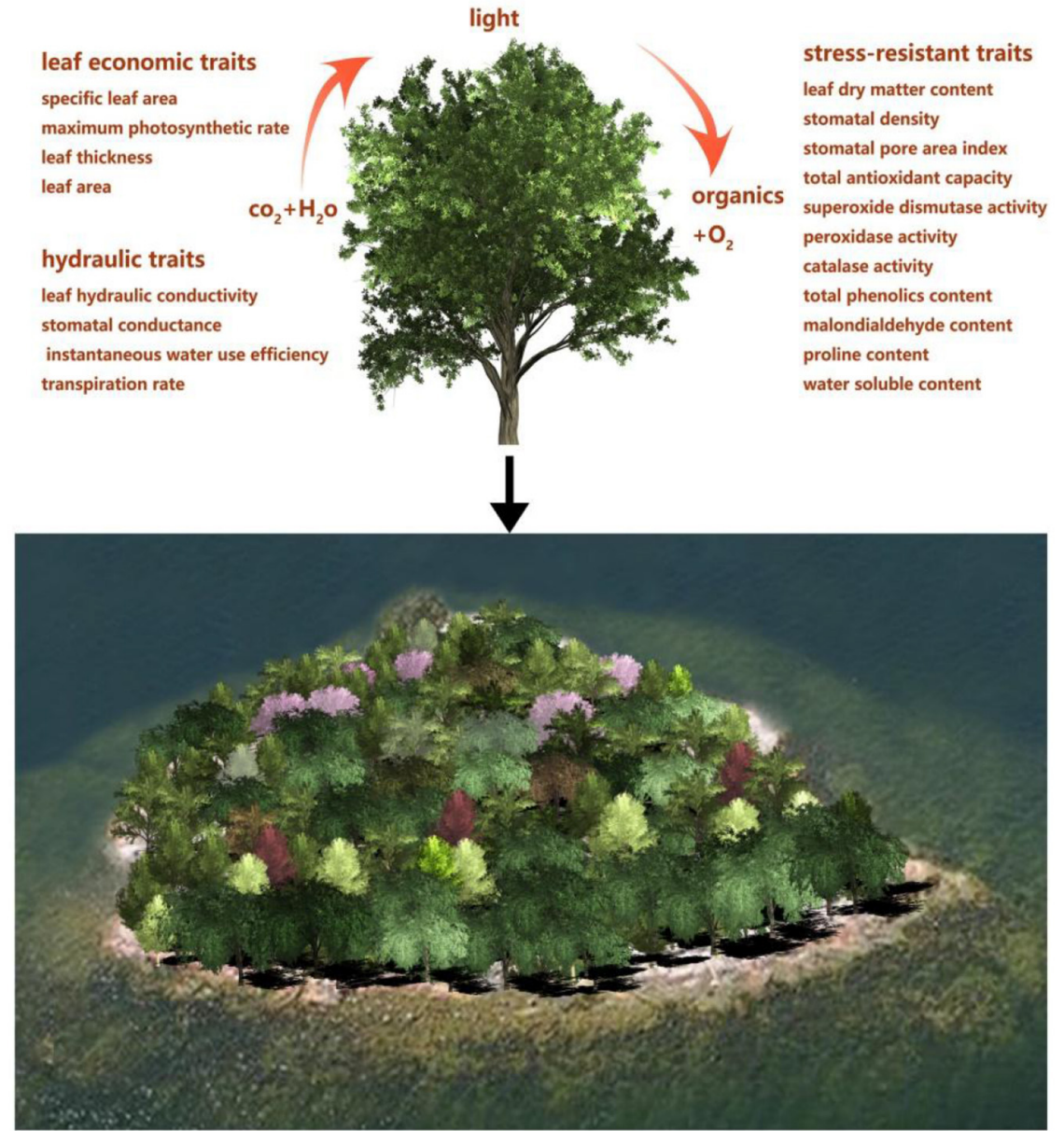

FIGURE 1 | A successful example for describing how to use the trait-based protocol developed by Wang et al. to quickly and effectively select many suitable native tree species to successfully restore an extremely degraded tropical coral island to tropical forest. 
extremely degraded coral island (Figure 1) and the invasion of Leucaena leucocephala.

Functional traits can also be used to evaluate the effectiveness and success of reforestation. In light of functional traits, the reforestation success of a reforestation project which is used to restore $0.2 \mathrm{~m}^{2}$ mining-induced extremely degraded tropical forest (merely consisting of bare rock) has been evaluated (Zhao et al.). Zhao et al. also pointed out the next step for this reforestation project should use trait-based protocol to select suitable late-successional native tree species to be mix-planted understory of the present tree species in this reforestation project. Through this, this extremely degraded tropical forest can be gradually restored to its originally undisturbed level. Similarly, functional traits can also facilitate the assessment of the influences of reforestation on soil water content and resistance to typhoon (Liu et al.; Li et al.). For example, Liu et al. has found reforestation based on mono-plantation of fast-growing nonnative tree species is unhelpful in recovering soil water content. That is because mono-plantation of fast-growing non-native tree species will trigger much higher soil water absorption than the native tree species. Finally they recommend a three-step method for recovering soil water content of extremely degraded tropical forest ecosystems via reforestation. First, using the slope, the deep soil layers of the secondary tropical forest should be used as a reference to reconstruct slope and soil layers of extremely degraded tropical forest. Then the same soils should be refilled from the secondary tropical forest to plant fast-growing tree species to minimize impacts from landslides and other soil disturbance events. Third, dominant slow-growing tree species from the adjacent secondary area should be planted within the fast-growing species stands to increase soil water content. In addition to measuring height and DBH (diameter at breast

\section{REFERENCES}

Brown, R. W., and Amacher, M. C. (1999). "Selecting plant species for ecological restoration: a perspective for land managers," in Revegetation With Native Species: Proceedings, 1997 Society for Ecological Restoration Annual Meeting, eds L. K. Holzworth and R. W. Brown (Fort Lauderdale, FL; Ogden, UT), $1-16$.

Crouzeilles, R., Curran, M., Ferreira, M. S., Lindenmayer, D. B., Grelle, C. E., and Benayas, J. M. R. (2016). A global meta-analysis on the ecological drivers of forest restoration success. Nat. Commun. 7, 1-8. doi: 10.1038/ncomms 11666

Crouzeilles, R., Ferreira, M. S., Chazdon, R. L., Lindenmayer, D. B., Sansevero, J. B., Monteiro, L., et al. (2017). Ecological restoration success is higher for natural regeneration than for active restoration in tropical forests. Sci. Adv. 3, e1701345. doi: $10.1126 /$ sciadv.1701345

Dobson, A. P., Bradshaw, A. D., and Baker, A. Á. (1997). Hopes for the future: restoration ecology and conservation biology. Science 277, 515-522. doi: $10.1126 /$ science.277.5325.515

Fry, E. L., Power, S. A., and Manning, P. (2014). Trait-based classification and manipulation of plant functional groups for biodiversity-ecosystem function experiments. J. Veget. Sci. 25, 248-261. doi: 10.1111/jvs.12068

Hobbs, R. J., and Harris, J. A. (2001). Restoration ecology: repairing the earth's ecosystems in the new millennium. Restorat. Ecol. 9, 239-246. doi: 10.1046/j.1526-100x.2001.009002239.x

Jones, T. A. (2013). Ecologically appropriate plant materials for restoration applications. Bioscience 63, 211-219. doi: 10.1525/bio.2013.63.3.9 height), Li et al. have found DBH of less than $5 \mathrm{~cm}$ and a height of less than $2 \mathrm{~m}$ are more prone to damage during severe weather events. In contrast, taller trees having a height greater than $20 \mathrm{~m}$ and a DBH of more than $20 \mathrm{~cm}$ faced minimal damage from typhoon Mangkhut. As a result, the restoration of natural forests that have the ability to withstand strong weather phenomena such as typhoons has been explored.

All in all, several new and key insights of trait-based principle and protocol which is used to guide how to use reforestation to recover degraded tropical forest have been achieved from our Research Topic. However, the generality and effectiveness of trait-based principle and protocol have to be tested in many other degraded tropical forests. We believe with many scientists participating in the trait-based reforestation principle and protocol research, trait-based effective and useful restoration protocol can be gradually attained.

\section{AUTHOR CONTRIBUTIONS}

HZ, GW, and WL organized and managed the Research Topic, did analysis of all published papers in the Research Topic. HZ wrote the paper. All authors contributed to the article and approved the submitted version.

\section{FUNDING}

This work was funded by the Specific Research Fund of the Innovation Platform for Academicians of Hainan Province (YSPTZX202017), Scientific Research Project of Ecological Restoration of Baopoling mountain in Sanya and High level talents of Hainan Natural Science Foundation (2019RC161).

Karina, O. S., Martha, B. M., Fabiola, L. B., and Cristina, M. G. (2021). Accelerating tropical cloud forest recovery: performance of nine late-successional tree species. Ecol. Eng. 166, 106237. doi: 10.1016/j.ecoleng.2021.106237

Lamb, D., Erskine, P. D., and Parrotta, J. A. (2005). Restoration of degraded tropical forest landscapes. Science 310, 1628-1632. doi: 10.1126/science.1111773

Martínez-Garza, C., and Howe, H. F. (2003). Restoring tropical diversity: beating the time tax on species loss. J. Appl. Ecol. 40, 423-429. doi: 10.1046/j.1365-2664.2003.00819.x

Mesquita, R. D. C. G., Massoca, P. E. D. S., Jakovac, C. C., Bentos, T. V., and Williamson, G. B. (2015). Amazon rain forest succession: stochasticity or land-use legacy? Bioscience 65, 849-861. doi: 10.1093/biosci/biv108

Ostertag, R., Warman, L., Cordell, S., and Vitousek, P. M. (2015). Using plant functional traits to restore Hawaiian rainforest. J. Appl. Ecol. 52, 805-809. doi: 10.1111/1365-2664.12413

Padilla, F. M., Ortega, R., Sánchez, J., and Pugnaire, F. I. (2009). Rethinking species selection for restoration of arid shrublands. Basic Appl. Ecol. 10, 640-647. doi: 10.1016/j.baae.2009.03.003

Reid, J. L., Fagan, M. E., and Zahawi, R. A. (2018). Positive site selection bias in meta-analyses comparing natural regeneration to active forest restoration. Sci. Adv. 4, eaas9143. doi: 10.1126/sciadv.aas9143

Rosenthal, G. (2003). Selecting target species to evaluate the success of wet grassland restoration. Agric. Ecosyst. Environ. 98, 227-246. doi: 10.1016/s0167-8809(03)00083-5

Shimamoto, C. Y., Padial, A. A., da Rosa, C. M., and Marques, M. C. (2018). Restoration of ecosystem services in tropical forests: a global meta-analysis. PLoS ONE 13, e0208523. doi: 10.1371/journal.pone.0208523 
Shoo, L. P., Freebody, K., Kanowski, J., and Catterall, C. P. (2016). Slow recovery of tropical old-field rainforest regrowth and the value and limitations of active restoration. Conserv. Biol. 30, 121-132. doi: 10.1111/cobi.12606

The Bonn Challenge (2011). United Nations Environment Programme (UNEP).

Tsuboike, Y., Fujii, M., Yoshida, Y., and Kamata, N. (2021). An indirect impact of Sika Deer overpopulation on eutrophication of an aquatic ecosystem via understory vegetation: an individual-based approach using nitrate reductase activity. Front. Ecol. Evol. 9, 626905. doi: 10.3389/fevo.2021.626905

UN Climate Summit (2014). New York Declaration on Forests.

Conflict of Interest: The authors declare that the research was conducted in the absence of any commercial or financial relationships that could be construed as a potential conflict of interest.
Publisher's Note: All claims expressed in this article are solely those of the authors and do not necessarily represent those of their affiliated organizations, or those of the publisher, the editors and the reviewers. Any product that may be evaluated in this article, or claim that may be made by its manufacturer, is not guaranteed or endorsed by the publisher.

Copyright (c) 2022 Zhang, Wang and Long. This is an open-access article distributed under the terms of the Creative Commons Attribution License (CC BY). The use, distribution or reproduction in other forums is permitted, provided the original author(s) and the copyright owner(s) are credited and that the original publication in this journal is cited, in accordance with accepted academic practice. No use, distribution or reproduction is permitted which does not comply with these terms. 